\title{
Novel Dithiolane-Based Ligands Combining Sigma and NMDA Receptor Interactions as Potential Neuroprotective Agents
}

\author{
Silvia Franchini, Pasquale Linciano, Giulia Puja, Annalisa Tait, Chiara Borsari, Nunzio Denora, \\ Rosa Maria Iacobazzi, Livio Brasili, and Claudia Sorbi*
}

Cite This: ACS Med. Chem. Lett. 2020, 11, 1028-1034

Read Online

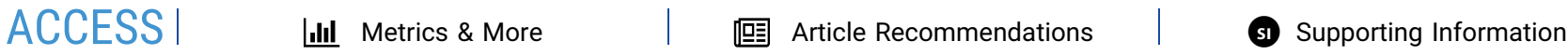

ABSTRACT: Sigma receptors (SRs) are recognized as valuable targets for the treatment of neurodegenerative disorders. A series of novel SRs ligands were designed by combining key pharmacophoric amines (i.e., benzylpiperidine or benzylpiperazine) with new 1,3-dithiolane-based heterocycles and their bioisosters. The new compounds exhibited a low nanomolar affinity for sigma-1 and sigma-2 receptors. Five selected compounds were evaluated for their neuroprotective capacity on SH-SY5Y neuroblastoma cell line. They were able to counteract

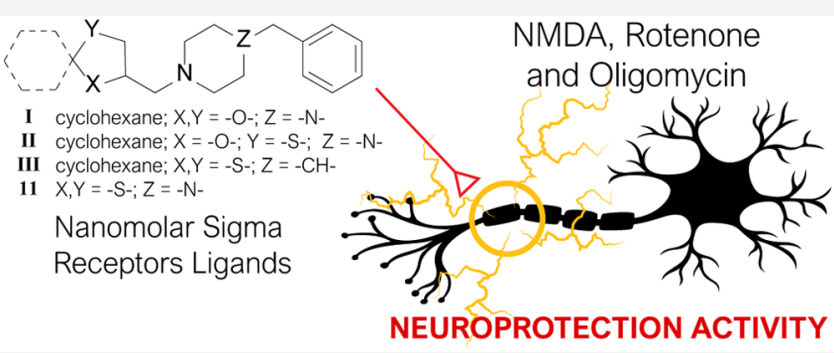
the neurotoxicity induced by rotenone, oligomycin and NMDA. Competition studies with PB212, a S1R antagonist, confirmed the involvement of S1R in neuroprotection from the oxidative stress induced by rotenone. Electrophysiological experiments performed on cortical neurons in culture highlighted the compounds ability to reduce NMDA-evoked currents, suggesting a negative allosteric modulator activity toward the NMDA receptor. Altogether these results qualify our novel dithiolane derivatives as potential agents for fighting neurodegeneration.

KEYWORDS: 1,3-Dithiolanes, neuroprotection, sigma receptors, NMDA, rotenone, neuroblastoma cancer cells SH-SY5Y

$\mathrm{N}$ eurodegeneration is a heterogeneous group of disorders including Alzheimer's disease (AD) and related dementia. According to the WHO around 50 million people worldwide are affected by dementia and nearly 10 million new cases are diagnosed every year. ${ }^{1}$ All drugs currently used in $\mathrm{AD}$ therapy are twenty-years old, have poor efficacy in preventing the progress of the disease, act exclusively on the symptoms, and present adverse side effects. ${ }^{2}$ Thus, there is an urgent need to identify novel targets and drugs for a disease-modifying therapy. The progressive loss of structure and/or function of neurons is the prototypical event behind neurodegeneration. ${ }^{3}$ Neurotoxicity induced by abnormal production of reactive oxygen species (ROS) is one the major causes of neuron loss and reflects an alteration of the mitochondrial respiratory chain activity. ${ }^{4}$ Oxidative cellular stress physiologically activates the sigma-1 receptor (S1R), a chaperone protein, mainly expressed in mitochondria-associated endoplasmic reticulum (ER) membranes. ${ }^{5}$ This receptor triggers or amplifies several cellular responses, including calcium homeostasis, reduction of glutamate release, ROS, nitric oxide (NO), microglial activity, and upregulation of antiapoptotic genes (i.e., Bcl-2), leading to neuroprotective effects. ${ }^{6-8}$ Indeed, S1R agonists have demonstrated neuroprotective properties using in vivo models of $\mathrm{AD}{ }^{9}$ In addition, increasing evidence suggests that modulating the sigma-2 receptor (S2R) can provide beneficial effects in neurodegenerative diseases. ${ }^{10}$ Among the numerous molecular targets that have been correlated with neurodegenerative disorders, also $N$-methyl-D-aspartate (NMDA) receptor plays a relevant role. ${ }^{11}$ A partnership between S1Rs and NMDA receptors leading to beneficial outcomes in neurodegeneration has been demonstrated. ${ }^{12}$ Over the last 10 years, our group has developed an extensive library of SR modulators combining different substituted five-membered heterocyclic rings with a broad range of amines (i.e., benzylpiperidine and benzylpiperazine).$^{13-15}$ Among the previously synthesized compounds, IIII (Table 1) were of particular interest. They behave as S1R agonists, with I and II showing high affinity for S1R $\left(K_{i}=0.74\right.$ and $1.3 \mathrm{nM}$, respectively) and moderate affinity for S2R subtype (S1R/S2R selectivity: 47 and 72, respectively), whereas compound III showed a low nanomolar affinity against both $\mathrm{SR}$ subtypes $\left(K_{\mathrm{i}} \mathrm{S} 1 \mathrm{R}=12 \mathrm{nM}\right.$ and $K_{\mathrm{i}} \mathrm{S} 2 \mathrm{R}=5.0$ $\mathrm{nM}) .{ }^{14}$ Molecular docking studies highlighted that a salt bridge between the protonated nitrogen atom of the benzylpiperazine/piperidine moiety and Glu172 is essential for the binding to S1R. ${ }^{16,17}$ The nature of the pharmacophoric amine (benzylpiperidine or benzylpiperazine) guided the binding mode within the S1R binding site. While the piperazine

Special Issue: In Memory of Maurizio Botta: His Vision of Medicinal Chemistry

Received: March 15, 2020

Accepted: April 3, 2020

Published: April 3, 2020

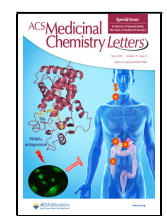


Table 1. Chemical Structure and Binding Affinity $\left(K_{i}\right.$ in $\left.\mathrm{nM}\right)$ for S1R and S2R of compounds I-III and 1-13

\begin{tabular}{|c|c|c|c|}
\hline Compd & Structure $^{d}$ & $\begin{array}{c}\text { S1R } \\
K_{i}(\mathrm{nM})^{\mathrm{a}, \mathrm{b}} \\
\end{array}$ & $\begin{array}{c}\mathbf{S}_{2} \mathbf{R} \\
K_{i}(\mathbf{n M})^{\mathrm{a}, \mathrm{c}} \\
\end{array}$ \\
\hline I & & 0.74 & 35 \\
\hline II & & 1.3 & 91 \\
\hline III & & 12 & 5.0 \\
\hline 1 & & 4.2 & 3.0 \\
\hline 2 & & 1.7 & 8.1 \\
\hline$(Z)-3$ & & 71 & 30 \\
\hline$(E)-3$ & & 107 & 65 \\
\hline$(Z)-4$ & & 129 & 93 \\
\hline$(E)-4$ & & 52 & 87 \\
\hline$(Z)-5$ & & 68 & 107 \\
\hline$(E)_{-5}$ & & 195 & 158 \\
\hline 6 & & 54 & 71 \\
\hline$(Z)-7$ & & 68 & 85 \\
\hline$(E)-7$ & & 76 & 63 \\
\hline 8 & & 2.0 & 0.59 \\
\hline 9 & & 0.50 & 12 \\
\hline 10 & & 0.60 & 3.3 \\
\hline 11 & & 2.1 & 90 \\
\hline 12 & & 0.60 & 6.2 \\
\hline 13 & & $5 \cdot 4$ & 182 \\
\hline
\end{tabular}

${ }^{a} \mathrm{SD}$ is within $\pm 10 \% .{ }^{b}$ Binding performed with $\left[{ }^{3} \mathrm{H}\right]$ pentazocine $3.0 \mathrm{nM} .{ }^{c}$ Binding performed with $\left[{ }^{3} \mathrm{H}\right]$ ditolylguanidine $3.0 \mathrm{nM}$. ${ }^{d}$ Compounds were tested as oxalate salts.

derivatives (i.e., I and II) positioned the spirocyclic moiety in the primary hydrophobic pocket delimitated by Met93, Tyr103, Ile 178, and Ala 185, the piperidine derivatives (i.e., III) exhibited a reverse docking mode with the benzyl moiety into the primary pocket (Figure $1 \mathrm{~A}$ and Figure SI-1A). ${ }^{14}$ To better investigate the structure activity relationships (SAR) of this SRs ligand series, herein we expanded the library by combining the pharmacophoric amines with dithiolane rings and their bioisosters. Considering the binding mode of compound III at S1R, 17 novel analogues were prepared modifying the spirocyclic moiety (Figure 1B) and aiming to clarify the steric and electrostatic properties necessary for strong interactions within the secondary hydrophobic pocket (compounds 1-9, Table 1). In accordance with our mixed ligand/structure-based investigation, the secondary hydrophobic pocket is able to accommodate bulky groups, as also proposed by Glennon's pharmacophoric model. ${ }^{14,18}$ In particular cycloaliphatic (pentane, eptane) and cycloaromatic groups (dihydro-indene, tetrahydro-naphtalene, benzo[7]annulene, fluorene) were introduced to progressively increase the structural rigidity. This strategy was successfully applied to haloperidol-constrained SR ligands providing compounds with enhanced affinity and selectivity for the S2R subtype. ${ }^{14}$ In addition the structural simplification of the spiro-portion was investigated in both benzylpiperazine and benzylpiperidine 
A

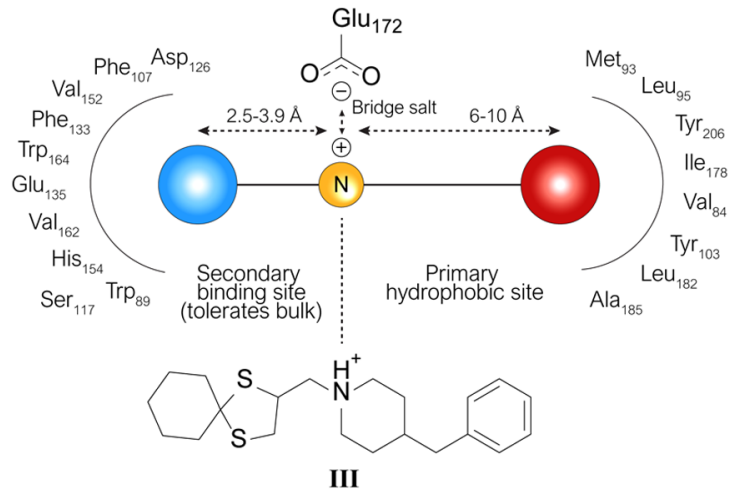

B

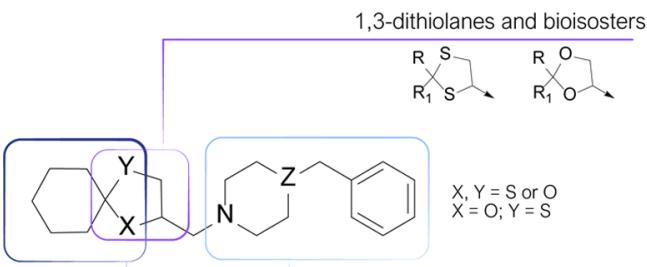

Modification of the spyro portion

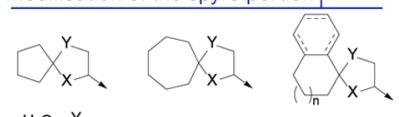

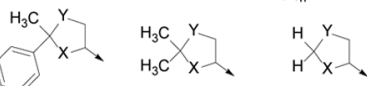

Figure 1. (A) Overview of the S1R binding site with the key Glu172 and the two main hydrophobic sites, and superimposition of Glennon's pharmacophore distances. The binding orientation of III, as representative for benzylpiperidine derivatives, predicted by docking calculations, is schematically shown. (B) Library design and expansion by combining the pharmacophoric amines with dithiolane rings and their bioisosters. series (compounds 8-11). The corresponding dioxolane bioisosters (12 and 13) were also prepared.

For the overall library, the most relevant physicochemical properties and drug-likeness were determined in silico using the SwissADME web utility. ${ }^{19}$ Prediction of blood-brain barrier (BBB) permeability has been pointed out as an important parameter in the design of neuroprotective compounds. Assuming no more than one violation to the Lipinski's rule of $5,{ }^{20}$ all the compounds showed a good druggability and were predicted to passively permeate the $\mathrm{BBB}$ (Table SI-1). The synthetic strategies for the synthesis of compounds 1-13 are described in Scheme 1. Compounds 113 are the oxalate salts obtained by reaction of the respective free amines 14-26 with anhydrous oxalic acid, followed by crystallization from dry diethyl ether. The free amines 14-26 were obtained by standard $\mathrm{S}_{\mathrm{N}} 2$ reaction between the appropriate aliphatic chloride 27-36 and 4-benzylpiperidine (for compounds 14-22, 25) or 1-benzylpiperazine (for 23, 24, and 26). The amines 16-18 and 20, characterized by the presence of two stereogenic centers, were obtained as a mixture of two diasteromeric pairs. The two racemates were separated by flash chromatography and the $E / Z$ isomerism assigned by NOESY experiments. With the exception of 36 , which was obtained directly by condensation of acetone with 3-chloro-1,2-propandiol, 4-(chloromethyl)-1,3-dithiolanes 2735 were prepared by treatment of the respective alcohols 3745 with thionyl chloride. Compounds $37-44$ were obtained by condensation of the appropriate ketone with 2,3-dimercaptopropan-1-ol using $\mathrm{HClO}_{4}$ absorbed on silica gel as catalyst. This procedure did not allow us to obtain 45 which was instead prepared by condensation of formaldehyde diethyl acetal with 2,3-dimercapto-propan-1-ol in the presence of cobalt chloride and trimethylsilyl chloride. All the synthesized compounds were evaluated for binding affinity toward S1R and

Scheme $1^{a}$
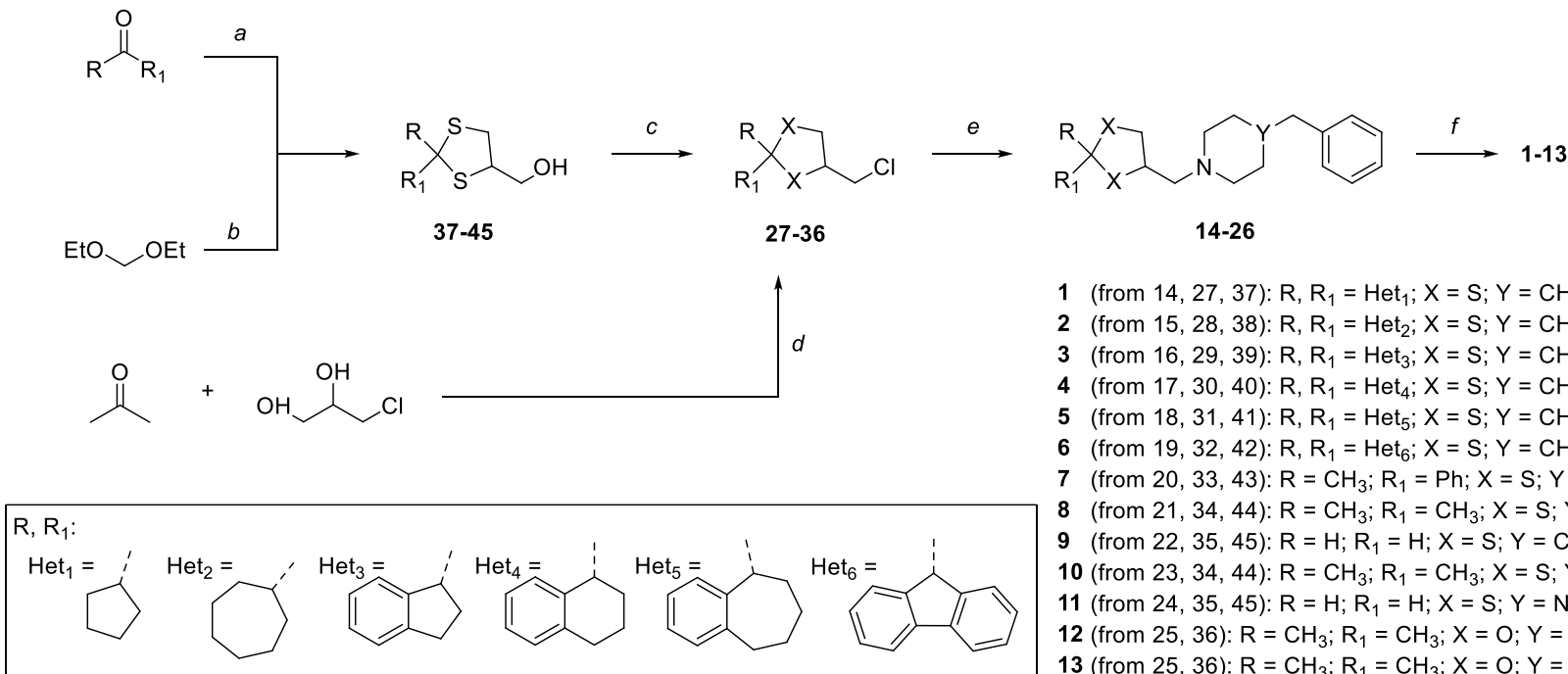

1 (from 14, 27, 37): R, R $\mathrm{R}_{1}=\mathrm{Het}_{1} ; \mathrm{X}=\mathrm{S} ; \mathrm{Y}=\mathrm{CH}$ 2 (from 15, 28, 38): R, $\mathrm{R}_{1}=\mathrm{Het}_{2} ; \mathrm{X}=\mathrm{S} ; \mathrm{Y}=\mathrm{CH}$ 3 (from 16, 29, 39): R, $\mathrm{R}_{1}=\mathrm{Het}_{3} ; \mathrm{X}=\mathrm{S} ; \mathrm{Y}=\mathrm{CH}$ 4 (from 17, 30, 40): R, $\mathrm{R}_{1}=\mathrm{Het}_{4} ; \mathrm{X}=\mathrm{S} ; \mathrm{Y}=\mathrm{CH}$ 5 (from 18, 31, 41): R, $\mathrm{R}_{1}=\mathrm{Het}_{5} ; \mathrm{X}=\mathrm{S} ; \mathrm{Y}=\mathrm{CH}$ 6 (from 19, 32, 42): $R, R_{1}=\mathrm{Het}_{6} ; X=S ; Y=C H$ 7 (from 20, 33, 43): $\mathrm{R}=\mathrm{CH}_{3} ; \mathrm{R}_{1}=\mathrm{Ph} ; \mathrm{X}=\mathrm{S} ; \mathrm{Y}=\mathrm{CH}$ 8 (from 21, 34, 44): $\mathrm{R}=\mathrm{CH}_{3} ; \mathrm{R}_{1}=\mathrm{CH}_{3} ; \mathrm{X}=\mathrm{S} ; \mathrm{Y}=\mathrm{CH}$ 9 (from 22, 35, 45): $\mathrm{R}=\mathrm{H} ; \mathrm{R}_{1}=\mathrm{H} ; \mathrm{X}=\mathrm{S} ; \mathrm{Y}=\mathrm{CH}$ 10 (from 23, 34, 44): $\mathrm{R}=\mathrm{CH}_{3} ; \mathrm{R}_{1}=\mathrm{CH}_{3} ; \mathrm{X}=\mathrm{S} ; \mathrm{Y}=\mathrm{N}$ 11 (from 24, 35, 45): $\mathrm{R}=\mathrm{H} ; \mathrm{R}_{1}=\mathrm{H} ; \mathrm{X}=\mathrm{S} ; \mathrm{Y}=\mathrm{N}$ 12 (from 25, 36): $\mathrm{R}=\mathrm{CH}_{3} ; \mathrm{R}_{1}=\mathrm{CH}_{3} ; \mathrm{X}=\mathrm{O} ; \mathrm{Y}=\mathrm{CH}$ 13 (from 25, 36): $\mathrm{R}=\mathrm{CH}_{3} ; \mathrm{R}_{1}=\mathrm{CH}_{3} ; \mathrm{X}=\mathrm{O} ; \mathrm{Y}=\mathrm{N}$

${ }^{a}$ Reagents and conditions: (a) appropriate ketone (1 equiv), 2,3-dimercapto-1-propanol (1.2-1.4 equiv), $\mathrm{HClO}_{4}$ on $\mathrm{SiO}_{2}(0.008-0.05$ equiv), neat, $\mathrm{N}_{2}$, MW irradiation, $50-80{ }^{\circ} \mathrm{C}, 15-60 \mathrm{~min}, 24-98 \%$ yield; (b) formaldehyde diethyl acetal (1 equiv), 2,3-dimercapto-1-propanol (1.2 equiv), $\mathrm{CoCl}_{2}$ (0.5 equiv), TMCS (0.8 equiv), dry acetonitrile, $\mathrm{N}_{2}$, r.t., $75 \mathrm{~min}, 24 \%$ yield; (c) thionyl chloride (1.2 equiv), DMF (cat.), dry dichloromethane, $\mathrm{N}_{2}, 0{ }^{\circ} \mathrm{C}, 90$ min, quant. yield; $69 \%$ (for 35); (d) acetone (10 equiv), 3-chloro-propan-1,2-diol (1 equiv), $\mathrm{HClO}_{4}$ on $\mathrm{SiO}_{2}(0.6$ equiv), dry dichloromethane, $\mathrm{N}_{2}, \mathrm{MW}$ irradiation, $80{ }^{\circ} \mathrm{C}, 100 \mathrm{~min}, 59 \%$ yield; (e) 4-benzylpiperidine or 1-benzylpiperazine $\left(2\right.$ equiv), $\mathrm{K}_{2} \mathrm{CO}_{3}(2$ equiv), KI (cat.), dry acetonitrile, MW irradiation, $130{ }^{\circ} \mathrm{C}, 10-40 \mathrm{~min}, 7-64 \%$ yield; (f) oxalic acid (1.2 equiv for piperidine or 2.2 equiv for piperazine), dry diethyl ether, r.t., 48 h, 32-90\% yield. 
S2R in a radioligand displacement assay using $\left[{ }^{3} \mathrm{H}\right]-(+)-$ pentazocine to selectively mark $\mathrm{S} 1 \mathrm{R}$ and $\left[{ }^{3} \mathrm{H}\right]$-ditolylguanidine, in the presence of cold (+)-pentazocine, to mark S2R. The binding affinities, expressed as inhibition constant $\left(K_{i}\right)$, are reported in Table 1 . The compounds showed nanomolar affinity at both SR subtypes with $K_{\mathrm{i}}$ values ranging from 195 to $0.5 \mathrm{nM}$ for S1R, and from 182 to $0.59 \mathrm{nM}$ for S2R. Compounds 9, 11, and 13 exhibited a moderate selectivity for S1R over S2R with a S1R/S2R ratio of 24,43 , and 34, respectively. The binding results, supported by docking calculation, allowed us to disclose some structure-activity relationships. The narrowing and expansion of the carbocyclic ring (1 and 2, respectively) preserved the high affinity of the lead compound III at both subtypes $\left(K_{\mathrm{i}} \mathrm{S} 1 \mathrm{R}=4.2\right.$ and $1.7 \mathrm{nM}$ and $K_{\mathrm{i}} \mathrm{S} 2 \mathrm{R}=3.0$ and $8.1 \mathrm{nM}$, respectively). Compounds 1 and 2 presented a binding mode analogue to that of compound III (Figure SI-1B). In contrast, scaffold rigidification obtained by condensing one (for compounds 3-5) or two (for 6) benzene rings caused a significant drop in affinity for both SR subtypes. Similarly, opening of the spirocyclic portion into the more flexible derivative 7 led to a reduced affinity at both subtypes $\left(K_{\mathrm{i}} \mathrm{S} 1 \mathrm{R}=68\right.$ and $76 \mathrm{nM}$ and $K_{\mathrm{i}} \mathrm{S} 2 \mathrm{R}=85$ and $63 \mathrm{nM}$ for $E$ and $Z$ isomers, respectively). These results highlighted that a bulky aromatic ring, condensed or appended to the 1,3-dithiolane core, is detrimental for affinity. As suggested by docking calculations, although the secondary binding site of S1R might tolerate bulky groups, the rigid and constrained conformation assumed by these molecules prevented an easy fit within the pocket. Thus, compounds 3-7 assumed the reverse binding mode, as previously observed for I and II.

The benzylpiperidine ring was located in the secondary site, while the bulkier spirocyclic moieties were accommodated in the wider and longer primary hydrophobic pocket. However, this led to an unsuitable alignment between the protonated piperidine nitrogen atom and the Glu172, justifying the reduced affinity of compounds 3-7 compared to III (Figure SI-1C). No diastereoselectivity between the $E / Z$ isomers was observed. The structural simplification of the 1,4-dithiaspirodecane moiety of III with a less sterically hindered and more flexible structure ( 8 and 9 ) resulted in a subnanomolar affinity $\left(K_{\mathrm{i}} \mathrm{S} 1 \mathrm{R}=2.0\right.$ and $0.59 \mathrm{nM}$ and $K_{\mathrm{i}} \mathrm{S} 2 \mathrm{R}=0.5$ and $12 \mathrm{nM}$, for 8 and 9, respectively). Compounds 8 and 9 showed a binding mode comparable to that described for III, 1-3 (Figure 2A). However, the opening of the cycloalkylspiro portion induced a slight shift of these compounds toward the secondary pocket, (i) straightening the salt bridge between the protonated amine and Glu172 and (ii) allowing the benzyl-tail to be engaged in an additional $\pi-\pi$ face-to-edge stacking with Tyr 103 .

The high S1R affinity was maintained by replacing the piperidine with the piperazine $\left(K_{\mathrm{i}}=0.6\right.$ and $2.1 \mathrm{nM}$ for 10 and 11, respectively). As expected, the presence of the benzylpiperazine reverted the binding poses of 10 and 11 in order to efficiently align the protonated nitrogen with Glu172 (Figure 2B). This binding mode fulfilled the Glennon's pharmacophore, preserving the distances of the two hydrophobic groups of the molecule to the central protonated site. Finally, no significant differences in terms of affinity and selectivity were observed by replacing the dithiolane (10 and 11) with the dioxolane bioisoster (12 and 13, Figure SI-1D). To sum up, the SAR investigation pointed out that (i) structural simplification of the spiro portion allowed achieving sub/low nanomolar affinity for SRs, while (ii) the introduction of bulky groups led to a drop in affinity for both SR subtypes.

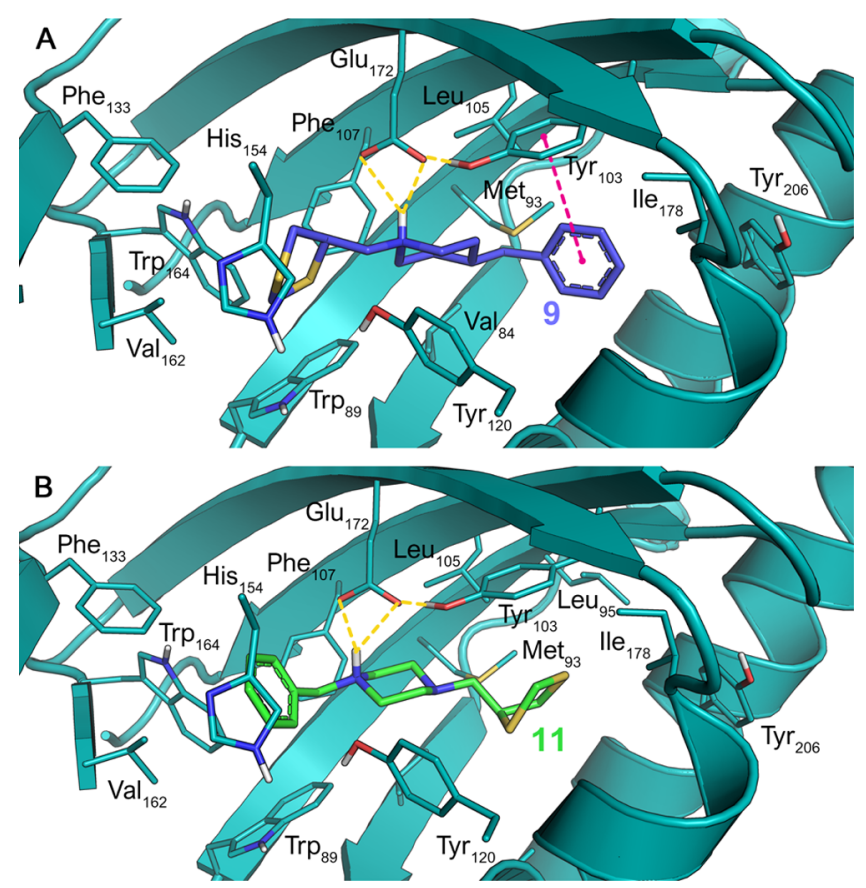

Figure 2. Docking of compound 9 (A, stick, violet carbon) and 11 (B, stick green carbon) into S1R binding site. The reference crystal structure used for the docking calculation (PDB ID: 6DK1) of S1R is shown in teal cartoon. Important interacting residues are in stick representation. Model atoms except for carbons are color-coded with protein carbons (teal), oxygen (red), nitrogen (blue), and sulfur (yellow). Bridge salt and $\pi-\pi$ interactions are represented as yellow and magenta dotted lines, respectively. Part of the $\beta$-barrel has been hidden for a clearer visualization of the binding site.

Recent studies suggested that both S1R and S2R modulators might have a positive effect in neuroprotection. ${ }^{5,10}$ Therefore, we evaluated the in vitro neuroprotective capacity of selected high affinity ligands. According to the binding affinities, compounds I, II, and 11 were chosen for their S1R selectivity, whereas III and 9 were selected as S1R/S2R mixed compounds. ROS are normally produced in neurons and the resulting oxidative stress is one of the major contributors to cell death in neurodegenerative disorders. Herein, we assessed the capability of compounds I-III, 9, and $\mathbf{1 1}$ to protect SHSY5Y cells from oxidative damage induced by two potent ROS generating insults, namely rotenone and oligomycin. ${ }^{21}$ At first, the selected compounds were investigated, as single agents, for cytotoxicity (Table SI-2) in human SH-SY5Y cell line to define the experimental doses for the neuroprotective studies. ${ }^{22}$ The neuroprotective capacity was reported as percentage of cell viability after treatment with the compound in the presence of the toxic stimuli.

At $1 \mu \mathrm{M}$ concentration, compounds I-III and $\mathbf{1 1}$ were able to significantly prevent cell damage induced by rotenone (Figure 3). Interestingly, I and II were effective also at the higher dose of $5 \mu \mathrm{M}$. To investigate the role of S1R in this process, the neuroprotective activity of our molecules was tested in the presence of PB212, a S1R antagonist. ${ }^{23}$ All compounds displayed a significant decrease of their neuroprotective capacity in combination with PB212 $(P<0,001$ at 1 $\mu \mathrm{M})$. Altogether these results suggested the involvement of sigma receptors in the neuroprotective effect of the proposed compounds. 


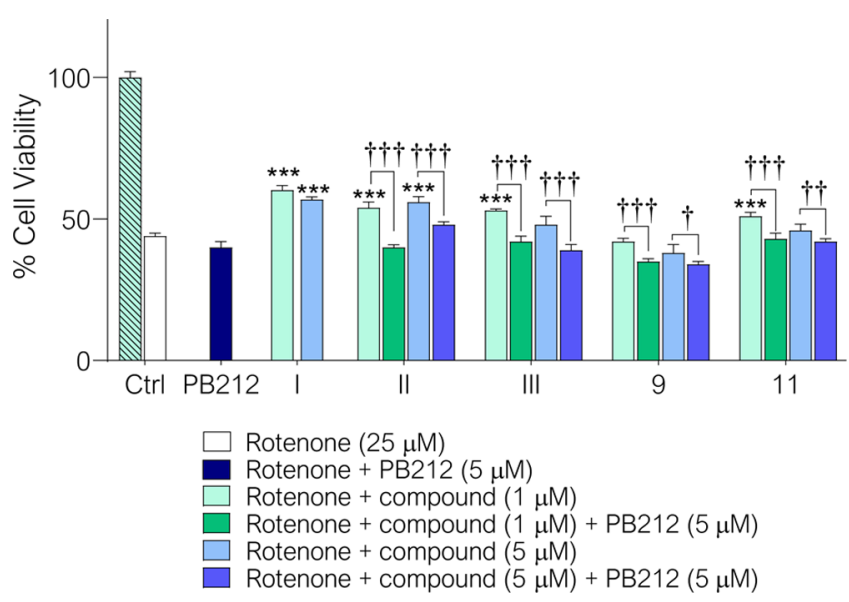

Figure 3. Neuroprotective effect, expressed as \% of cell viability (SHSY5Y cells) of the tested compounds I-III, 9, and 11 at 1.0 and 5.0 $\mu \mathrm{M}$ in the presence of rotenone $(25 \mu \mathrm{M})$ and a combination of rotenone and the S1R antagonist, PB212 $(5 \mu \mathrm{M})$. Each bar represents the mean \pm SD of three independent experiments: $* * * p<0.001$ vs rotenone alone, ${ }^{\dagger \dagger \dagger} p<0.001,{ }^{\dagger \dagger} p<0.01$, and ${ }^{\dagger} p<0.05$.

When oligomycin $(2.5 \mu \mathrm{M})$ was used as a toxic stimulus, only compound II at $5 \mu \mathrm{M}$ was able to protect cells from death, with a cell viability $>60 \%$ (Figure SI-2). In addition, the neuroprotective potential of our ligands was evaluated using NMDA as a toxic insult. ${ }^{24}$ The neuroblastoma cells were incubated for $24 \mathrm{~h}$ in the presence of $250 \mu \mathrm{M}$ of NMDA (corresponding to its $\mathrm{IC}_{50}$, Table SI-2) and four different concentrations of compounds I-III, 9 , and 11 (ranging from 0.1 to $5 \mu \mathrm{M}$ ). Memantine was used as a positive control at the same concentrations of the tested molecules. ${ }^{25}$ All the tested compounds demonstrated a neuroprotective effect against NMDA stimuli at concentrations $<1 \mu \mathrm{M}$ (Figure 4). I, III, and

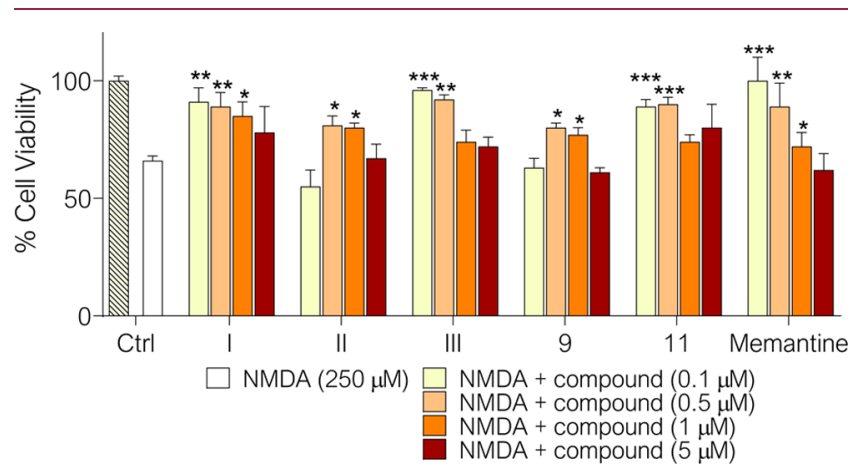

Figure 4. Neuroprotective effect, expressed as \% of cell viability (SHSY5Y cells) of the tested compounds I-III, 9, and 11 at $0.1,0.5,1.0$, and $5.0 \mu \mathrm{M}$ in the presence of NMDA $250 \mu \mathrm{M}\left(\mathrm{IC}_{50}\right.$ at $\left.24 \mathrm{~h}\right)$. Memantine was used as positive control. Each bar represents the mean \pm SD of three independent experiments: $*_{p}<0.05$, ** $p<0.01$, $* * * p<0.001$ vs NMDA alone.

11 showed the highest neuroprotective activity, being able to recover $>85 \%$ of the cell viability at the two lowest doses $(0.1$ and $0.5 \mu \mathrm{M})$. Moreover, I and III exhibited a dose-response profile similar to that of memantine. Compounds II and 9 showed a lower cytoprotective activity compared to I, III, and $11(72-75 \%$ of the cell survival at 0.5 and $1 \mu \mathrm{M})$. All tested compounds, including memantine, did not significantly increase cell viability at a $5 \mu \mathrm{M}$ concentration.
Compounds III and 11, showing the best neuroprotective activity, were selected to further demonstrate the involvement of SRs in their neuroprotective effects against NMDA toxicity. In the presence of PB212, the neuroprotective activity of both compounds (tested at $0.1 \mu \mathrm{M})$ significantly decreased $(P<$ $0,001)$, even though it was not completely abolished for compound III (Figure 5).

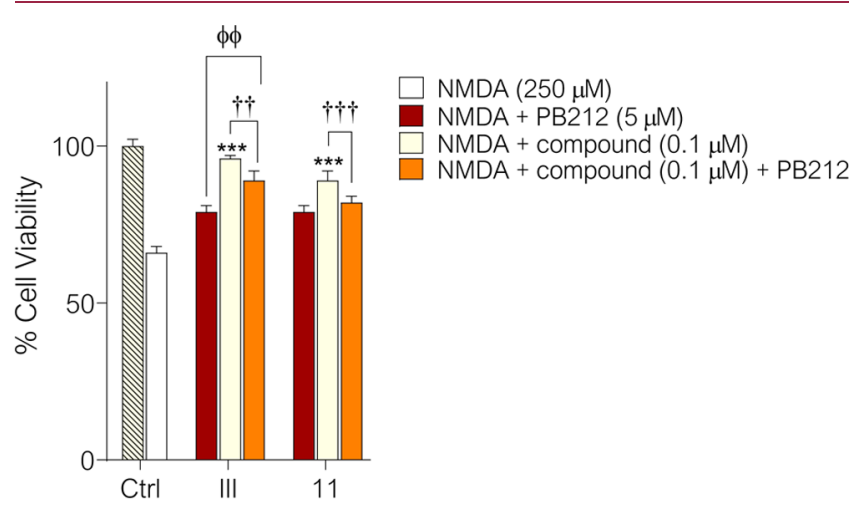

Figure 5. Neuroprotective effect, expressed as \% of cell viability (SHSY5Y cells), of the tested compounds III and $11(0.1 \mu \mathrm{M})$ in the presence of NMDA $(250 \mu \mathrm{M})$ and a combination of NMDA and PB212 $(5 \mu \mathrm{M})$. Each bar represents the mean \pm SD of three independent experiments: $* * * p<0.001$ vs NMDA alone, ${ }^{\dagger \dagger} p<$ 0.001 and ${ }^{\dagger \dagger} p<0.01,{ }^{\varphi \varphi \varphi} p<0.001$.

In the literature, examples of SR ligands with promiscuous affinity for NMDA receptors are reported; thus, the neuroprotective capacity of III could be related to its activity on NMDA receptors. ${ }^{12}$ To test this hypothesis, we used a patch clamp technique in primary culture of cortical neurons and investigated the ability of compounds I-III to modulate NMDA-evoked current. $^{26}$ Compound I reduced NMDAevoked currents of 34 and $18 \%$ at a concentration of 0.1 and $1 \mu \mathrm{M}$, respectively (Figure 6).

Similarly, low concentrations of compounds II and III decreased NMDA-evoked currents $(-20 \pm 4 \%$ for II and -50 $\pm 6 \%$ for III), suggesting that the neuroprotection exerted by these compounds against NMDA-induced cytotoxicity could be achieved through a reduction of NMDA receptor-mediated ion flux. On the contrary, compound I at $10 \mu \mathrm{M}$ increased (110\%) NMDA current (Figure 6). This effect could explain the lack of neuroprotection of $\mathbf{I}$ at high concentrations (Figure 4). These data support the hypothesis that compounds I-III, in addition to interacting with the sigma receptors, can also act as negative allosteric modulators of NMDA receptors.

In summary, we identified novel high affinity ligands and assessed their neuroprotective effects. Compounds II and $\mathbf{1 1}$ displayed an excellent affinity for S1R $\left(K_{i}=1.3\right.$ and 2.1) and selectivity over S2R ( 72 and 43 , respectively). II was able to protect neuronal cells from three different cytotoxic insults, namely rotenone, oligomycin, and NMDA. In the case of rotenone, the neuroprotective activity of the tested compounds was completely reversed after treatment with PB212, a S1R antagonist, highlighting that its effect is fully mediated by SRs. This is consistent with a S1R agonist activity. ${ }^{14}$ However, for NMDA induced toxicity, PB212 did not completely abolish the neuroprotective capacity of III, suggesting that its effect could be mediated by an either direct or indirect modulation of the NMDA receptor. Interestingly, compounds bearing the 4benzylpiperazine basic moiety (I, II, and 11) displayed strong 

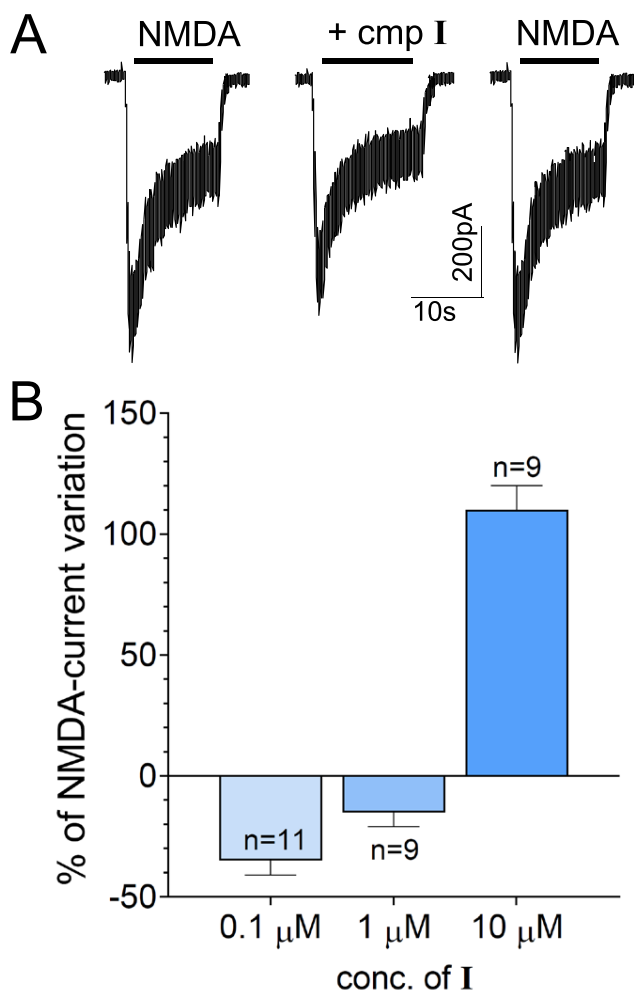

Figure 6. Modulation of NMDA-evoked currents by compound I. (A) Representative electrophysiological traces showing the effect of I (1 $\mu \mathrm{M})$ on the current evoked by NMDA $100 \mu \mathrm{M}+$ glycine $1 \mu \mathrm{M}$ (NMDA) in a cortical neuron grown in primary culture. Bars show the duration of drug application. (B) Histogram showing the effect (express as a \% of the control current) of increasing concentrations of I on NMDA-evoked currents. Each bar is the mean \pm SE of $n$ cells tested.

S1R affinities together with in vitro neuroprotection, with II being the best one. Altogether, our results encourage the further evaluation of these spirocyclic derivatives in animal models of $\mathrm{AD}$.

\section{ASSOCIATED CONTENT}

\section{(s) Supporting Information}

The Supporting Information is available free of charge at https://pubs.acs.org/doi/10.1021/acsmedchemlett.0c00129.

Experimental Section: (i) synthetic procedures and characterization of compounds 1-13; (ii) binding assay for SRs; (iii) molecular docking; (iv) cellular assays for neuroprotection activity; Figure SI-1, Predicted binding mode of III, 1, 4, and 12; Figure SI-2, Neuroprotection activity in the presence of oligomycin; Table SI-1, Predicted physicochemical properties, drug-likeness, and blood-brain barrier permeability; Table SI-2, cytotoxicity of compounds I-III, 9, and 11, memantine, and NMDA in SH-SY5Y cell lines. (PDF)

\section{AUTHOR INFORMATION}

\section{Corresponding Author}

Claudia Sorbi - Department of Life Sciences, University of Modena and Reggio Emilia, 41125 Modena, Italy; (ㄱ) orcid.org/0000-0001-6916-4933; Phone: +39 59205 8583; Email: claudia.sorbi@unimore.it

\section{Authors}

Silvia Franchini - Department of Life Sciences, University of Modena and Reggio Emilia, 41125 Modena, Italy; (1) orcid.org/0000-0002-6320-9712

Pasquale Linciano - Department of Life Sciences, University of Modena and Reggio Emilia, 41125 Modena, Italy; (1) orcid.org/0000-0003-0382-7479

Giulia Puja - Department of Life Sciences, University of Modena and Reggio Emilia, 41125 Modena, Italy; (1) orcid.org/00000001-8385-6020

Annalisa Tait - Department of Life Sciences, University of Modena and Reggio Emilia, 41125 Modena, Italy; (1) orcid.org/0000-0002-4105-5409

Chiara Borsari - Department of Life Sciences, University of Modena and Reggio Emilia, 41125 Modena, Italy; (1) orcid.org/0000-0002-4688-8362

Nunzio Denora - Dipartimento di Farmacia - Scienze del Farmaco, Università degli Studi di Bari Aldo Moro, 70126 Bari, Italy; ○ orcid.org/0000-0002-7756-7828

Rosa Maria Iacobazzi - IRCCS Istituto tumori "Giovanni Paolo II", 70124 Bari, Italy; @ orcid.org/0000-0002-07376591

Livio Brasili - Department of Life Sciences, University of Modena and Reggio Emilia, 41125 Modena, Italy; (1) orcid.org/0000-0002-3280-3196

Complete contact information is available at: https://pubs.acs.org/10.1021/acsmedchemlett.0c00129

\section{Author Contributions}

CS: conceived the work, performed the synthesis and the data analysis; SF: performed the synthesis and the data analysis; PL and AT: performed the structural characterization and the data analysis; PL: performed molecular docking and SAR analysis; $\mathrm{CB}$ : performed the synthesis and structural characterization; GP: performed the electrophysiological studies; ND and RMI: performed the neuroprotection studies; LB: conceived and supervised the work. The manuscript was written through contributions of all authors. All authors have given approval to the final version of the manuscript.

\section{Funding}

This research was supported by the University of Modena and Reggio Emilia (FAR 2017- ITACA Project and FAR JUNIOR 2018- SMILE Project).

\section{Notes}

The authors declare no competing financial interest.

\section{ACKNOWLEDGMENTS}

The authors thank the National Institute of Mental Health's Psychoactive Drug Screening Program (NIMH-PDSP) for the sigma receptor binding data generously provided (Contract \# HHSN-271-2018-00023-C) and used in this paper. The S1R antagonist, PB212, was kindly provided by Carmen Abate, Department of Pharmacy - Pharmaceutical Sciences of the University of Bari, Italy.

\section{ABBREVIATIONS}

$\mathrm{AD}$, Alzheimer's disease; NMDA, N-methyl-(D)-aspartate; NO, nitric oxide; ROS, reactive oxygen species; SR, sigma receptor; $\mathrm{S} 1 \mathrm{R}$, sigma-1 receptor; $\mathrm{S} 2 \mathrm{R}$, sigma-2 receptor 


\section{REFERENCES}

(1) Patterson, C. World Alzheimer Report 2018. The State of the Art of Dementia Research: New Frontiers; Alzheimer's Disease International (ADI): London, 2018; pp 3-46.

(2) Briggs, R.; Kennelly, S. P.; O’Neill, D. Drug Treatments in Alzheimer's Disease. Clin. Med. 2016, 16 (3), 247-253.

(3) Chi, H.; Chang, H.-Y.; Sang, T.-K. Neuronal Cell Death Mechanisms in Major Neurodegenerative Diseases. Int. J. Mol. Sci. 2018, 19 (10), 3082.

(4) Guo, C.; Sun, L.; Chen, X.; Zhang, D. Oxidative Stress, Mitochondrial Damage and Neurodegenerative Diseases. Neural Regen. Res. 2013, 8 (21), 2003-2014.

(5) Smith, S. B. Introduction to Sigma Receptors: Their Role in Disease and as Therapeutic Targets. Adv. Exp. Med. Biol. 2017, 964, $1-4$.

(6) Collina, S.; Rui, M.; Stotani, S.; Bignardi, E.; Rossi, D.; Curti, D.; Giordanetto, F.; Malacrida, A.; Scuteri, A.; Cavaletti, G. Are Sigma Receptor Modulators a Weapon against Multiple Sclerosis Disease? Future Med. Chem. 2017, 9 (17), 2029-2051.

(7) Nguyen, L.; Lucke-Wold, B. P.; Mookerjee, S. A.; Cavendish, J. Z.; Robson, M. J.; Scandinaro, A. L.; Matsumoto, R. R. Role of Sigma1 Receptors in Neurodegenerative Diseases. J. Pharmacol. Sci. 2015, 127 (1), 17-29.

(8) Nguyen, L.; Kaushal, N.; Robson, M. J.; Matsumoto, R. R. Sigma Receptors as Potential Therapeutic Targets for Neuroprotection. Eur. J. Pharmacol. 2014, 743, 42-47.

(9) Maurice, T.; Volle, J.-N.; Strehaiano, M.; Crouzier, L.; Pereira, C.; Kaloyanov, N.; Virieux, D.; Pirat, J.-L. Neuroprotection in NonTransgenic and Transgenic Mouse Models of Alzheimer's Disease by Positive Modulation of Sigmal Receptors. Pharmacol. Res. 2019, 144, $315-330$.

(10) Abate, C.; Niso, M.; Berardi, F. Sigma-2 Receptor: Past, Present and Perspectives on Multiple Therapeutic Exploitations. Future Med. Chem. 2018, 10 (16), 1997-2018.

(11) Lau, A.; Tymianski, M. Glutamate Receptors, Neurotoxicity and Neurodegeneration. Pfluegers Arch. 2010, 460 (2), 525-542.

(12) Pabba, M.; Sibille, E. Sigma-1 and N-Methyl-D-Aspartate Receptors: A Partnership with Beneficial Outcomes. Mol. Neuropsychiatry 2015, 1 (1), 47-51.

(13) Franchini, S.; Battisti, U. M.; Prandi, A.; Tait, A.; Borsari, C.; Cichero, E.; Fossa, P.; Cilia, A.; Prezzavento, O.; Ronsisvalle, S.; et al. Scouting New Sigma Receptor Ligands: Synthesis, Pharmacological Evaluation and Molecular Modeling of 1,3-Dioxolane-Based Structures and Derivatives. Eur. J. Med. Chem. 2016, 112, 1-19.

(14) Franchini, S.; Sorbi, C.; Battisti, U. M.; Tait, A.; Bencheva, L. I.; Cichero, E.; Fossa, P.; Cilia, A.; Prezzavento, O.; Ronsisvalle, S.; et al. Structure-Activity Relationships within a Series of $A_{1}$ and ${ }_{2}$ Receptor Ligands: Identification of $\mathrm{a}_{2}$ Receptor Agonist (BS148) with Selective Toxicity against Metastatic Melanoma. ChemMedChem 2017, 12 (22), 1893-1905.

(15) Costantino, L.; Gandolfi, F.; Sorbi, C.; Franchini, S.; Prezzavento, O.; Vittorio, F.; Ronsisvalle, G.; Leonardi, A.; Poggesi, E.; Brasili, L. Synthesis and Structure-Activity Relationships of 1Aralkyl-4- Benzylpiperidine and 1-Aralkyl-4-Benzylpiperazine Derivatives as Potent $\sigma$ Ligands. J. Med. Chem. 2005, 48 (1), 266-273.

(16) Ablordeppey, S. Y.; Fischer, J. B.; Glennon, R. A. Is a Nitrogen Atom an Important Pharmacophoric Element in Sigma Ligand Binding? Bioorg. Med. Chem. 2000, 8 (8), 2105-2111.

(17) Ramachandran, S.; Chu, U. B.; Mavlyutov, T. A.; Pal, A.; Pyne, S.; Ruoho, A. E. The Sigma1 Receptor Interacts with N-Alkyl Amines and Endogenous Sphingolipids. Eur. J. Pharmacol. 2009, 609 (1-3), 19-26.

(18) Glennon, R. A.; Ablordeppey, S. Y.; Ismaiel, A. M.; ElAshmawy, M. B.; Fischer, J. B.; Howie, K. B. Structural Features Important for $\sigma_{1}$ Receptor Binding. J. Med. Chem. 1994, 37 (8), 1214-1219.

(19) Daina, A.; Michielin, O.; Zoete, V. SwissADME: A Free Web Tool to Evaluate Pharmacokinetics, Drug-Likeness and Medicinal
Chemistry Friendliness of Small Molecules. Sci. Rep. 2017, 7 (January), 1-13.

(20) Lipinski, C. A. Drug-like Properties and the Causes of Poor Solubility and Poor Permeability. J. Pharmacol. Toxicol. Methods 2000, 44 (1), 235-249.

(21) Franchini, S.; Bencheva, L. I.; Battisti, U. M.; Tait, A.; Sorbi, C.; Fossa, P.; Cichero, E.; Ronsisvalle, S.; Aricò, G.; Denora, N.; et al. Synthesis and Biological Evaluation of 1,3-Dioxolane-Based 5- $\mathrm{HT}_{1 \mathrm{~A}}$ Receptor Agonists for CNS Disorders and Neuropathic Pain. Future Med. Chem. 2018, 10 (18), 2137

(22) Ault, D. T.; Werling, L. L. SH-SY5Y Cells as a Model for Sigma Receptor Regulation of Potassium-Stimulated Dopamine Release. Brain Res. 2000, 877 (2), 354-360.

(23) Gasparre, G.; Abate, C.; Berardi, F.; Cassano, G. The Sigma-1 Receptor Antagonist PB212 Reduces the $\mathrm{Ca}^{2+}$-Release through the Inositol (1, 4, 5)-Trisphosphate Receptor in SK-N-SH Cells. Eur. J. Pharmacol. 2012, 684, 59.

(24) De Candia, M.; Zaetta, G.; Denora, N.; Tricarico, D.; Majellaro, M.; Cellamare, S.; Altomare, C. D. New Azepino[4,3-b]Indole Derivatives as Nanomolar Selective Inhibitors of Human Butyrylcholinesterase Showing Protective Effects against NMDA-Induced Neurotoxicity. Eur. J. Med. Chem. 2017, 125, 288-298.

(25) Geldenhuys, W. J.; Darvesh, A. S. Pharmacotherapy of Alzheimer's Disease: Current and Future Trends. Expert Rev. Neurother. 2015, 15 (1), 3-5.

(26) Hamill, O. P.; Marty, A.; Neher, E.; Sakmann, B.; Sigworth, F. J. Improved Patch-Clamp Techniques for High-Resolution Current Recording from Cells and Cell-Free Membrane Patches. Pfluegers Arch. 1981, 391 (2), 85-100. 